\title{
Finite centroid and helical axis estimation from noisy landmark measurements in the study of human joint kinematics
}

Citation for published version (APA):

Woltring, H. J., Huiskes, H. W. J., Lange, de, A., \& Veldpaus, F. E. (1985). Finite centroid and helical axis estimation from noisy landmark measurements in the study of human joint kinematics. Journal of Biomechanics, 18(5), 379-389. https://doi.org/10.1016/0021-9290(85)90293-3

DOI:

10.1016/0021-9290(85)90293-3

Document status and date:

Published: 01/01/1985

\section{Document Version:}

Publisher's PDF, also known as Version of Record (includes final page, issue and volume numbers)

\section{Please check the document version of this publication:}

- A submitted manuscript is the version of the article upon submission and before peer-review. There can be important differences between the submitted version and the official published version of record. People interested in the research are advised to contact the author for the final version of the publication, or visit the $\mathrm{DOI}$ to the publisher's website.

- The final author version and the galley proof are versions of the publication after peer review.

- The final published version features the final layout of the paper including the volume, issue and page numbers.

Link to publication

\section{General rights}

Copyright and moral rights for the publications made accessible in the public portal are retained by the authors and/or other copyright owners and it is a condition of accessing publications that users recognise and abide by the legal requirements associated with these rights.

- Users may download and print one copy of any publication from the public portal for the purpose of private study or research.

- You may not further distribute the material or use it for any profit-making activity or commercial gain

- You may freely distribute the URL identifying the publication in the public portal.

If the publication is distributed under the terms of Article 25fa of the Dutch Copyright Act, indicated by the "Taverne" license above, please follow below link for the End User Agreement:

www.tue.nl/taverne

Take down policy

If you believe that this document breaches copyright please contact us at:

openaccess@tue.nl

providing details and we will investigate your claim. 


\title{
FINITE CENTROID AND HELICAL AXIS ESTIMATION FROM NOISY LANDMARK MEASUREMENTS IN THE STUDY OF HUMAN JOINT KINEMATICS
}

\author{
H. J. Woltring†, R. Huiskes and A. DE LANGE \\ Biomechanics Section, Laboratory for Experimental Orthopaedics, Faculty of Medicine and Dentistry, \\ University of Nijmegen, P.O.Box 9101, NL-6500 HB Nijmegen, The Netherlands \\ and

\section{F. E. Veldpaus 2} \\ Department of Mechanical Engineering, Technical University Eindhoven, P.O. Box 513, NL-5600 MB \\ Eindhoven, The Netherlands
}

\begin{abstract}
Recent work on joint kinematics indicates that the finite centroid (centre of rotation) and the finite helical axis (axis of rotation, screw axis, twist axis) are highly susceptible to measurement errors when they are experimentally determined from landmark position data. This paper presents an analytical model to describe these effects, under isotropic conditions for the measurement errors and for the spatial landmark distribution. It appears that the position and direction errors are inversely proportional to the rotation magnitude, and that they are much more error-prone than the relatively well-determined rotation and translation magnitudes. Furthermore, the direction and rotation magnitude errors are inversely proportional to the landmark distribution radius, and the position and translation magnitude errors are minimal if the mean position of the landmarks coincides with the centroid or helical axis. For the planar centroid, the use of rigid-body constraints results in considerable precision improvement relative to the classical, finite Reuleaux method for centroid reconstruction.

These analytical results can be used to define suitable measurement configurations, and they are used in this paper to explain experimental results on Röntgenphotogrammetrically acquired, in vitro wrist joint movement.
\end{abstract}

\section{INTRODUCTION}

The study of joint function is an important aspect of orthopaedics and rehabilitation medicine, in view of the social and economic costs of joint disorders caused by trauma or arthritis. Such studies should be objective and repeatable, in order to allow within- and betweensubject comparisons, and this necessitates unambiguous, quantitative descriptions of joint movement. For this reason, the biomechanics literature is replete with publications on planar and three-dimensional joint kinematics; see, for example, Panjabi, 1979; Panjabi et al., 1982a, b; Soudan et al., 1979; Huiskes et al., in press; Bryant et al., 1984.

Next to position vectors and to attitude matrices or Euler/Cardan angles, the use of the helical axis (also called twist axis, screw axis, or axis of rotation) in the three-dimensional case, and of the centroid (also called centre of rotation) in the planar case has been quite common for joint movement quantification and visualization. At each moment in time, a continuously moving rigid body (e.g. a bone) may be viewed as

Received December 1983; in revised form 12 December 1984 * Parts of this work were presented at the A.S.M.E. Symposium 'Biomechanics 1983', Houston, TX, June 1983. † Present address: Philips Medical Systems, SWE-CAR, Building QA-2, P.O.B. 218, NL-5600 MD Eindhoven, The Netherlands. having a translation (or shift) velocity along, and a rotation velocity about a directed line in space (Fig. 1). The position of this helical axis will generally vary during the movement, and the movement is completely known once the translation and rotation velocities, and the position and direction of the helical axis, are known over time. In the planar case, the helical axis reduces to a single point, and only the rotation velocity and the centroid position are retained as movement quantifiers.

One should note that the helical axis and centroid are differential quantities, unlike the position vector and attitude matrix. This explains some of the experimental problems which are apparent from the literature. In particular, most users have approximated the instantaneous helical axis and centroid by means of the

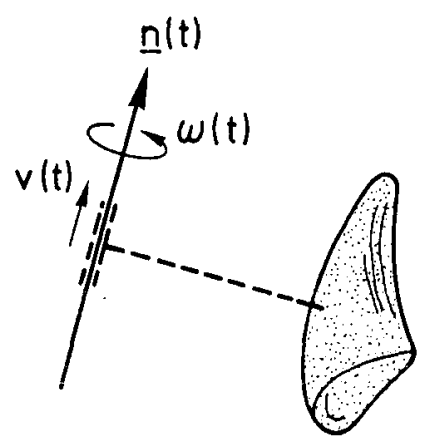

Fig. 1. Instantaneous helical axis. 
so-called finite helical axis and centroid which are estimated from single, finite displacements (Fig. 2). It appears that stochastic errors in the position and direction of the finite helical axis and centroid are inversely proportional to the finite rotation magnitude; however, small increments are required in order to reliably approximate the continuous movement by means of a sequence of finite displacements. Thus, it is necessary to find a reasonable balance between these deterministic and stochastic, conflicting error sources.

It is unfortunate that the terms instantaneous axis and centre of rotation are often used to denote the finite axis and centre of rotation. In the present paper, the term finite refers to a truly finite displacement, while the term instantaneous refers to the limiting case of a vanishing displacement, such as when decreasing the sampling interval in digitized, continuous movement.

The paper deals with statistically optimal estimation procedures for the finite helical axis and centroid as determined from noisy landmark position data, and with the effect of measurement errors on the estimated quantities. Important issues in this context are: (1) the incorporation of rigid-body constraints in the mathematical models, (2) the use of statistically optimal weighting schemes, so that the measurements are used in proportion to their accuracy, and (3) the minimization of statistical biases by avoiding strong nonlinear transformations before noise reduction through linear processing of redundant measurements. It will be shown that various methods reported in the literature fail in these respects.

Under analytically tractable assumptions (isotropic measurement errors and isotropic, spatial landmark distributions), the dependence of the errors in the helical and centroid estimates on geometrical and

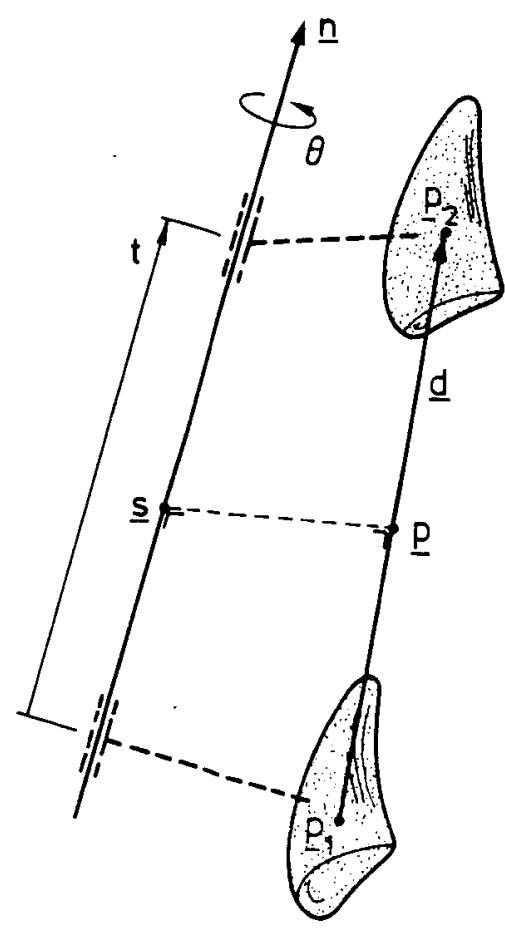

Fig. 2. Finite helical axis. kinematical quantities, and on the measurement error statistics is derived. A simplified analysis of the finite helical axis has recently been provided by Spoor (1984), who validated his results by means of a Monte-Carlo simulation study, and who also provided an illustration by means of experimental, tarsal joint motion data. In the present paper, the analytical results are illustrated by means of experimental data on wrist joint motion.

Since the finite centroid and helical axis are unacceptably noisy in practical situations, a summary indication is included on the use of continuous movement models through proper low-pass smoothing and differentiation procedures, in order to improve the accuracy of estimators for the true, instantaneous centroid and helical axis.

\section{THE SPATIAL CASE: HELICAL AXIS}

We assume a rigid body applied with a right-handed, cartesian co-ordinate system $E^{x}$ to undergo a displacement from a position and attitude $i=1$ to a position and attitude $i=2$ (Fig. 3). The body has a given distribution of $m \geqslant 3$ landmarks with known coordinates $\left\{x_{k}\right\}$ in $E^{x}(k=1, \ldots, m)$, at least three of which should be non-colinear for determinacy in rigidbody calculus to obtain. For reasons of analytical convenience, the origin of $E^{x}$ is chosen to coincide with the mean position $\mathbf{x}$ of the landmarks, and a landmark distribution matrix $A$ is defined from the landmark positions with respect to $\mathbf{x}$ (the symbol 'denotes transposition),

$$
\mathbf{x} \hat{=} \frac{1}{m} \sum_{k=1}^{m} \mathbf{x}_{k}=\mathbf{0} ; \quad A \hat{=} \operatorname{trace}(K) I-K
$$

where

$$
K \stackrel{1}{m} \sum_{k=1}^{m}\left(\mathbf{x}_{k}-\mathbf{x}\right)\left(\mathbf{x}_{k}-\mathbf{x}\right)^{\prime}=\frac{1}{m} \sum_{k=1}^{m} \mathbf{x}_{k} \mathbf{x}_{k}^{\prime} .
$$

The matrix A plays a key role in error propagation analysis for direction and rotation errors. If $K$ is

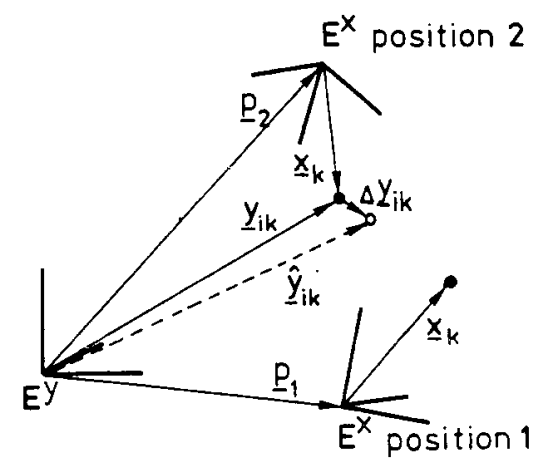

Fig. 3. Global (fixed) co-ordinate system $E^{y}$ in which a rigid body with attached co-ordinate system $E^{x}$ moves from position 1 to position 2 . The body has a given landmark distribution with known co-ordinates $\left\{\mathbf{x}_{k}\right\}$ in $E^{x}$, and true coordinates $\left\{\mathbf{y}_{i k}\right\}$ in $E^{y}$, with $i=1,2, k=1 \ldots m \geqslant 3$. Due to measurement errors $\left\{\Delta \mathbf{y}_{i k}\right\}$, only observed co-ordinates $\left\{\hat{\mathbf{y}}_{i k}\right\}$ 
proportional to the identity matrix, so is $A$, and the landmark distribution is called isotropic,

$$
A=\rho^{2} I ; \quad \rho^{2}=\frac{2}{3} r^{2} ; \quad K=\frac{1}{3} r^{2} I
$$

where $\rho$ is the effective landmark distribution radius. It is the r.m.s. distance of the landmarks to an arbitrary line through $\mathbf{x}$, while $r$ is the r.m.s. distance of the landmarks to $\mathbf{x}$ proper. Examples of isotropic landmark distributions are those where the landmarks are at the vertices of a regular polyhedron, such as a regular triangular pyramid $(m=4)$ or a cube $(m=8)$.

\section{Position and attitude}

The two positions of the origin of $E^{x}$ with respect to a global (external), right-handed cartesian co-ordinate system $E^{y}$ are denoted by the vector $\mathrm{p}_{i}, i=1,2$, and the attitudes of $E^{x}$ with respect to $E^{y}$ by the attitude matrix $R_{i}$, where $R_{i}$ is orthonormal, with determinant +1 .

By means of some experimental procedure, estimated values $\left\{\hat{\mathbf{y}}_{i k}\right\}$ for the landmarks' true coordinates $\left\{\mathbf{y}_{i k}\right\}$ in $E^{y}$ can be obtained. For error-free $\left\{\hat{\mathbf{y}}_{i k}\right\}$, i.e., $\hat{\mathbf{y}}_{i k}=\mathbf{y}_{i k}$, the $\mathrm{p}_{i}$ and $R_{i}$ can subsequently be determined from the rigid-body model

$$
\mathbf{y}_{i k}=\mathbf{p}_{i}+R_{i} \mathbf{x}_{k} ; \quad R_{i}^{\prime} R_{i}=I ; \quad i=1,2 ; k=1, \ldots, m
$$

using a least-squares algorithm such as described by Spoor and Veldpaus (1980) or Veldpaus et al. (in press). If the $\left\{\mathbf{y}_{i k}\right\}$ are additively disturbed by zero-mean, uncorrelated, isotropic errors with constant standard deviation $\sigma$ per co-ordinate,

$$
\hat{\mathbf{y}}_{i k} \hat{=} \mathbf{y}_{i k}+\Delta \mathbf{y}_{i k} ; E\left(\Delta \mathbf{y}_{i k}\right)=0 ; E\left(\Delta \mathbf{y}_{i k} \Delta \mathbf{y}_{j l}^{\prime}\right)=\delta_{i j} \delta_{k l} \sigma^{2} I
$$

where $E$ denotes the expectancy operator, and $\delta_{i j}$ the Kronecker delta $\left(\delta_{i j}=1\right.$ if $i=j, \delta_{i j}=0$ if $\left.i \neq j\right)$, leastsquares algorithms provide estimates $\hat{\mathbf{p}}_{i}$ and $\hat{R}_{i}$ which are statistically optimal in the minimum variance (or Gauss-Markov) sense. For the theoretical part of this paper, the model (4) is entertained. However, one may note that practical error distributions often do not meet this model. For arbitrary error covariance matrices, unweighted least-squares algorithms do not provide minimum variance estimators; it may then prove useful to resort to some form of generalized adjustment calculus (cf. Mikhail, 1976; Woltring, 1980, 1982).

In order to create a linear error propagation model, the relation between the estimates $\hat{\mathbf{p}}_{i}, \hat{R}_{i}$ and the underlying true values $\mathbf{p}_{i}, R_{i}$ is modelled additively for the $\hat{\mathbf{p}}_{i}$, and multiplicatively for the $\hat{R}_{i}$,

$$
\hat{\mathbf{p}}_{i} \hat{=} \mathbf{p}_{i}+\Delta \mathbf{p}_{i} ; \quad \hat{R}_{i} \hat{=}\left(I H+\Delta R_{i}\right) R_{i}
$$

where $I+\Delta R_{i}$ is an orthonormal matrix, with determinant +1 . Thus, $\left(I+\Delta R_{i}\right)^{\prime}\left(I+\Delta R_{i}\right)=I$, whence we find that $\Delta R_{i}+\Delta R_{i}^{\prime}+\Delta R_{i}^{\prime} \Delta R_{i}=0$. For sufficiently small attitude errors, the term $\Delta R_{i}^{\prime} \Delta R_{i}$ can be neglected, and we find that $\Delta R_{i}+\Delta R_{i}^{\prime}=0$. Thus, $\Delta R_{i}$ is a skew-symmetric (or anti-symmetric) matrix, for sufficiently small measurement errors.
A skew-symmetric matrix $S \hat{=} S\{\mathbf{a}\}$ can be characterized in terms of its axial vector $\mathbf{a}$,

$$
S\{\mathbf{a}\} \hat{=}\left[\begin{array}{ccc}
0 & -a_{z} & a_{y} \\
a_{z} & 0 & -a_{x} \\
-a_{y} & a_{x} & 0
\end{array}\right] ; \mathbf{a} \hat{=}\left[\begin{array}{l}
a_{x} \\
a_{y} \\
a_{z}
\end{array}\right]
$$

with the following properties,

$$
S\{\mathbf{a}\}=-S\{\mathbf{a}\}^{\prime} ; S\{\mathbf{a}\} S\{\mathbf{b}\}=\mathbf{b a}^{\prime}-\mathbf{a}^{\prime} \mathbf{b} I
$$

$S\{\mathbf{a}\} \mathbf{b}=-S\{\mathbf{b}\} \mathbf{a}=\mathbf{a}^{*} \mathbf{b}=-\mathbf{b}^{*} \mathbf{a}$ (for arbitrary $\mathbf{a}, \mathbf{b}$ )

and where ${ }^{*}$ denotes the vector product operator. It follows from (6-7) that the matrix $\Delta R_{i}$ in (5) can be expressed in terms of a small attitude error vector $\Delta \phi_{i}$,

$$
\Delta R_{i}=S\left\{\Delta \phi_{i}\right\} ; \quad\left|\Delta \phi_{i}\right| \ll 1 \mathrm{rad} .
$$

The components of $\Delta \mathbf{p}_{i}$ represent translations along the co-ordinate axes of $E^{y}$, and those of $\Delta \phi_{i}$ small, incremental rotations about these axes. Under the smallness condition (8), these rotations behave as commuting vector components.

Separation of the 'noise' equations from (3-4) results in a linear error propagation model for the rigid-body errors $\Delta \mathbf{p}_{i}$ and $\Delta \phi_{i}$,

$$
B_{i k} \Delta \mathbf{u}_{i}=\Delta \mathbf{y}_{i k}
$$

where

$$
B_{i k} \hat{=}\left(I, S^{\prime}\left\{R_{i} \mathbf{x}_{k}\right\}\right) ; \quad \Delta \mathbf{u}_{i} \hat{=}\left(\Delta \mathbf{p}_{i}^{\prime}, \Delta \phi_{i}^{\prime}\right)^{\prime} .
$$

It follows that the rigid-body errors are unbiased, since the measurement errors are unbiased by assumption, and since unbiasedness is maintained under a linear transformation. Conventional error propagation calculus (Mikhail, 1976) then provides the covariance matrix of $\Delta \mathbf{u}_{i}$ as

$$
E\left(\Delta \mathbf{u}_{i} \Delta \mathbf{u}_{j}^{\prime}\right)=\delta_{i j} \sigma^{2} B_{i}^{-1} ; \quad B_{i} \hat{=} \sum_{k=1}^{m} B_{i k}^{\prime} B_{i k} .
$$

Under the given choice $\mathbf{x}=\mathbf{0}$ (1a) this results in uncorrelated position and attitude errors,

$$
\begin{aligned}
E\left(\Delta \mathbf{p}_{i} \Delta \mathbf{p}_{j}^{\prime}\right) & =\delta_{i j} \frac{\sigma^{2}}{m} I \\
E\left(\Delta \mathbf{p}_{i} \Delta \phi_{j}^{\prime}\right) & =0 \\
E\left(\Delta \phi_{i} \Delta \phi_{j}^{\prime}\right) & =\delta_{i j} \frac{\sigma^{2}}{m} R_{i} A^{-1} R_{i}^{\prime}
\end{aligned}
$$

where $A$ is the landmark distribution matrix defined in (1a). For the case of an isotropic landmark distribution (2), relation (11c) reduces to

$$
E\left(\Delta \phi_{i} \Delta \phi_{j}^{\prime}\right)=\delta_{i j} \frac{\sigma^{2}}{m \rho^{2}} I .
$$

Thus, the attitude errors are generally anisotropic for arbitrary $A$ and $R_{i}$, but they become isotropic if the landmark distribution is isotropic. Furthermore, the position errors are always isotropic under the present error model.

\section{Helical axis parameters}

The finite helical axis representing a displacement $\left(\mathbf{p}_{1} \rightarrow \mathbf{p}_{2} ; R_{1} \rightarrow R_{2}\right)$ in $E^{y}$ can be characterized in terms 
of a unit direction vector $\mathbf{n}$ and by the position $\mathbf{s}$ of some point on this axis. The finite rotation angle about the axis is denoted with $\theta$, and the finite translation or shift along this axis with $t$ (see Fig. 2). Following Spoor and Veldpaus (1980), $\mathbf{n}$ and $\theta$ can be derived from the relations

$$
\begin{aligned}
\cos \theta I+(1-\cos \theta) \mathbf{n n}^{\prime} & =\frac{1}{2}\left(R+R^{\prime}\right) \\
\sin \theta S\{\mathbf{n}\} & =\frac{1}{2}\left(R-R^{\prime}\right)
\end{aligned}
$$

with

$$
R \hat{=} R_{2} R_{1}^{\prime} ; \quad \mathbf{n}^{\prime} \mathbf{n} \hat{=} 1 ; \quad 0<\theta \leqslant \pi \mathrm{rad}
$$

and $S\{$.$\} the skew-symmetric matrix defined in (6).$ Spoor and Veldpaus (1980) selected for $\mathbf{s}$ the projection of the origin of $E^{y}$ onto the helical axis; however, it has been found more advantageous to use the mean value of the origin of $E^{x}$ for this purpose. Thus, $\mathbf{s}$ is chosen to be the projection onto the helical axis of the midpoint $p$ on the finite translation vector $\mathbf{d}$ from $\mathbf{p}_{1}$ to $\mathbf{p}_{2}$,

$$
\mathbf{p} \hat{=} \frac{1}{2}\left(\mathbf{p}_{1}+\mathbf{p}_{2}\right) ; \quad \mathbf{d} \hat{=} \mathbf{p}_{2}-\mathbf{p}_{1} .
$$

From the projective condition $\mathbf{n}^{\prime}(\mathbf{s}-\mathbf{p})=0, \mathbf{s}$ and $t$ follow as (cf. Spoor and Veldpaus, 1980),

$$
\mathbf{s}=\mathbf{p}+\left\{2 \tan \left(\frac{1}{2} \theta\right)\right\}^{-1} \mathbf{n}^{*} \mathbf{d} ; \quad t=\mathbf{n}^{\prime} \mathbf{d} .
$$

Since $\mathbf{s}$ is the projection of $\mathbf{p}$ onto the helical axis, $\mathbf{s}-\mathbf{p}$ is the shortest line between the landmarks' mean position (averaged over $i=1,2$ ) and the helical axis. It is possible to prove that $\mathbf{s}$ has the highest precision of all points on the helical axis, under the conditions (2) and (4).

The helical axis is undefined for $\theta=0$, i.e. under a pure translation. This explains the extreme sensitivity of the finite helical axis to measurement errors under small rotations. For very large rotations, Spoor and Veldpaus (1980) suggested using relation (12a) since $\sin \theta$ in (12b) then becomes too small. However, this situation is unlikely for the case that the finite helical axis is used to approximate the true, instantaneous helical axis.

The helical variables can be estimated by substituting the estimates $\hat{\mathrm{p}}_{i}$ and $\hat{R}_{i}$ for the true values $\mathrm{p}_{i}$ and $R_{i}$ in (12-14). In this fashion, the measurement errors in the $\left\{\hat{\mathbf{y}}_{i k}\right\}$ are averaged out before the strongly nonlinear transformations (12-14), and this reduces the influence of non-linearity biases. Conversely, strong non-linear transformations on measurements with additive, zero-mean errors before some form of linear averaging may result in relatively strong biases in the eventually estimated quantities. For example, it is relatively easy to estimate individual helical parameters from three non-collinear landmarks, but the use of an average helical axis from a linear combination of all possible axes, for $m>3$, would be statistically unsound. This will be discussed more fully for some published models of the planar centroid.

\section{Helical error propagation analysis}

The helical errors can be analysed via linearized error propagation as before. Thus, the estimated and true parameters are modelled to be related as

$$
\begin{array}{ll}
\hat{\theta}=\theta+\Delta \theta ; & \hat{\mathbf{n}} \hat{=} \mathbf{n}+\Delta \mathbf{n} \\
\hat{t}=\mathrm{t}+\Delta t ; & \hat{\mathbf{s}}=\mathbf{s}+\Delta \mathbf{s} .
\end{array}
$$

Under the linearity assumption for sufficiently small measurement errors, unbiasedness is maintained, and the covariance matrices of the helical errors follow via the partial derivatives of these errors to the rigid-body errors $\Delta \mathrm{p}_{i}$ and $\Delta \phi_{i}$. The partial derivatives can be evaluated at the expected error values, i.e. for the true values $\mathbf{p}_{i}$ and $R_{i}$. As a starting point, the covariance matrices of $\Delta \mathbf{p}$ and $\Delta \mathrm{d}$ in

$$
\hat{\mathbf{p}} \hat{=} \mathbf{p}+\Delta \mathbf{p} ; \quad \hat{\mathbf{d}} \hat{=} \mathbf{d}+\Delta \mathbf{d}
$$

can be evaluated as

$$
\begin{gathered}
E\left(\Delta \mathbf{p} \Delta \mathbf{p}^{\prime}\right)=\frac{\sigma^{2}}{2 m} I ; \quad E\left(\Delta \mathbf{p} \Delta \mathbf{d}^{\prime}\right)=0 ; \\
E\left(\Delta \mathbf{d} \Delta \mathbf{d}^{\prime}\right)=\frac{2 \sigma^{2}}{m} I .
\end{gathered}
$$

Furthermore, it has proved useful to define intermediate rotation vectors $\hat{\chi}$ and $\chi$ as

$\hat{\chi} \hat{=} \chi+\Delta \chi ; \quad S\{\hat{\chi}\} \hat{=} \frac{1}{2}\left(\hat{R}-\hat{R}^{\prime}\right) ; \quad S\{\chi\} \hat{=} \frac{1}{2}\left(R-R^{\prime}\right)$.

This can be combined with (5), (8) and (12) to yield the following linear relation between $\Delta \chi$ and the $\Delta \phi_{i}$,

$$
\begin{aligned}
\Delta \chi= & \frac{1}{2}\{\operatorname{trace}(R) I-R] \Delta \phi_{2} \\
& -\frac{1}{2}\left\{\operatorname{trace}(R) I-R^{\prime}\right\} \Delta \phi_{1} .
\end{aligned}
$$

For arbitrary landmark distribution matrix $A$ (1), combination of (11c) and (19) results in a rather awkward expression for $E\left(\Delta \chi \Delta \chi^{\prime}\right)$. However, for the isotropic case (2), combination with (11d) results in

$$
E\left(\Delta \chi \Delta \chi^{\prime}\right)=\frac{\sigma^{2}}{m \rho^{2}}\left\{(1+\cos \theta)\left(I-\mathbf{n n}^{\prime}\right)+2 \cos ^{2} \theta \mathbf{n n}^{\prime}\right\} .
$$

The rotation and direction estimates are merely a function of $\hat{\chi}$, not of $\hat{\mathbf{p}}$ or $\hat{\boldsymbol{d}}$. Their partial derivatives to the rotation error vector $\Delta \chi$ follow from (12), (15), (18), and (19) as

$$
\partial \hat{\boldsymbol{\theta}} / \partial \Delta \chi=\mathbf{n}^{\prime} / \cos \theta ; \quad \partial \hat{\mathbf{n}} / \partial \Delta \chi=\sin ^{-1} \theta\left(I-\mathbf{n n}^{\prime}\right)
$$

and linear covariance propagation with (20-21) yields

$$
\begin{aligned}
\sigma_{\hat{\theta}}^{2} \hat{=} E\left\{(\Delta \theta)^{2}\right\} & =\frac{2 \sigma^{2}}{m \rho^{2}} \\
\Sigma_{\hat{\mathbf{n}}} \hat{=} E\left(\Delta \mathbf{n} \Delta \mathbf{n}^{\prime}\right) & =\sigma_{\hat{\theta}}^{2}\left\{2 \sin \left(\frac{1}{2} \theta\right)\right\}^{-2}\left(I-\mathbf{n n}^{\prime}\right) .
\end{aligned}
$$

Since $\Delta \mathrm{p}_{i}$ and $\Delta \phi_{i}$ are uncorrelated, $\Delta \phi_{i}$ is uncorrelated with $\Delta \mathrm{p}$ and $\Delta \mathrm{d}$. Furthermore, since $\Delta \chi$ is a linear function of the $\Delta \phi_{i}$, and since also $\Delta p$ and $\Delta d$ are uncorrelated, the variance of $\hat{t}$ and the covariance matrix of $\hat{\mathbf{s}}$ follow by adding the contributions due to $\Delta \mathbf{p}, \Delta \mathrm{d}$, and $\Delta \chi$. After extensive but straightforward 
matrix calculus, this results in

$$
\sigma_{t}^{2} \hat{=} E\left\{(\Delta t)^{2}\right\}=\frac{2 \sigma^{2}}{m}\left(1+\cos ^{-2}\left(\frac{1}{2} \theta\right)|\mathbf{s}-\mathbf{p}|^{2} / \rho^{2}\right)
$$

and a rather complex relation for $E\left(\Delta \mathbf{s} \Delta \mathbf{s}^{\prime}\right)$. However, only the error components normal to $\mathbf{n}$ are relevant, since any point on the helical axis may be used for determining the axis' position. After applying a projection operator $\left(I-\mathbf{n n}^{\prime}\right)$ to this covariance matrix in order to remove the variance components parallel to $\mathbf{n}$,

$\Sigma_{\mathbf{s} \perp \mathbf{n}} \hat{=} E_{\lfloor\mathbf{n}}\left(\Delta \mathbf{s} \Delta \mathbf{s}^{\prime}\right)=\left(I-\mathbf{n} \mathbf{n}^{\prime}\right) E\left(\Delta \mathbf{s} \Delta \mathbf{s}^{\prime}\right)\left(I-\mathbf{n n}^{\prime}\right)(24)$

the total variances of the spatial parameters $\hat{\mathbf{n}}$ and $\hat{\mathbf{s}}$ over all relevant directions follow from the traces of the respective covariance matrices. This results in

$$
\begin{aligned}
\sigma_{\hat{\mathrm{n}}}^{2} \hat{=} & \operatorname{trace}\left(\Sigma_{\hat{\mathbf{n}}}\right)=2 \sigma_{\hat{\partial}}^{2} /\left\{2 \sin \left(\frac{1}{2} \theta\right)\right\}^{2} \\
\sigma_{\hat{\mathrm{s}} \mid \mathrm{n}}^{2} \hat{=} & \operatorname{trace}\left(\Sigma_{\hat{\mathrm{s}} \mid \mathbf{n}}\right) \\
= & \frac{\sigma^{2}}{m} \sin ^{-2}\left(\frac{1}{2} \theta\right)\left(1+\frac{1}{4} \tan ^{-2}\left(\frac{1}{2} \theta\right) t^{2} / \rho^{2}\right. \\
& \left.+\frac{1}{2} \cos ^{-2}\left(\frac{1}{2} \theta\right)|\mathbf{s}-\mathbf{p}|^{2} / \rho^{2}\right) .
\end{aligned}
$$

If the finite helical axis is used to approximate the true, instantaneous helical axis, one may assume that $|\theta| \ll 1 \mathrm{rad}$; under this assumption, the variances in the isotropic landmark distribution case reduce to

$\sigma_{\hat{\theta}}^{2}=\frac{2 \sigma^{2}}{m \rho^{2}} ; \quad \sigma_{\hat{\mathrm{n}}}^{2} \simeq 2 \sigma_{\hat{\theta}}^{2} / \theta^{2} ; \quad \sigma_{\hat{t}}^{2} \simeq \frac{2 \sigma^{2}}{m}\left\{1+|\mathbf{s}-\mathbf{p}|^{2} / \rho^{2}\right\}$

$$
\sigma_{\hat{\mathbf{s}} \perp \mathbf{n}}^{2} \simeq \frac{\sigma^{2}}{m \theta^{2}}\left(4+\rho^{-2}\left\{4 \frac{t^{2}}{\theta^{2}}+2|\mathbf{s}-\mathbf{p}|^{2}\right\}\right) .
$$

For small values $\sigma_{\hat{\mathbf{n}}} \ll 1$, this quantity denotes the r.m.s. value of the angle $\alpha_{\hat{\mathbf{n}}}$ between the estimated and true direction vectors (in radians); cf. Fig. 4.

It appears from (22) that the rotation errors do not depend on the magnitude $\theta$ of the rotation angle. For small $\theta$, (26) shows that also the shift errors are invariant with $\theta$, while the position and direction errors vary inversely with $\theta$. The latter is, of course, related to the fact that the helical axis becomes undefined for

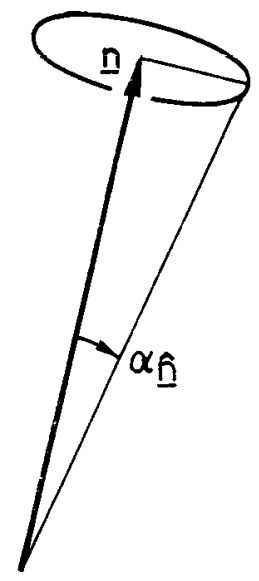

Fig. 4. Angular uncertainty $\alpha_{\hat{\mathbf{n}}}$ in the direction vector $\hat{\mathbf{n}}$. $\theta=0$. Furthermore, the position error in (26) increases with the ratio of the shift $t$ and the 'effective rotational translation' $\theta \rho$ of the landmarks, while the shift and position errors are minimal if the helical axis coincides with the centre of the landmark distribution, i.e. if $\mathbf{s}=\mathbf{p}$. Moreover, the rotation and direction errors are inversely proportional to the effective landmark distribution radius $\rho$. Thus, it is advantageous to select the landmark distribution in such a fashion that these factors have a sufficiently small influence on the $a$ posteriori error levels. In particular, the helical axis should always be close to the landmark distribution, and $\rho$ should be sufficiently large.

A numerical example may illustrate these points. In wrist kinematics, joint flexion excursion may be of the order of $100^{\circ}$, so the choice $\theta=0.1 \mathrm{rad} \simeq 5.7^{\circ}$ will allow approximately seventeen finite steps. The dimensions of the carpal bones are such that $\rho=5 \mathrm{~mm}$ is about the maximal value possible, and $\sigma=25 \mu \mathrm{m}$ is a realistic value when using Röntgenphotogrammetry (note however, that neither the error distribution nor the landmark distribution are isotropic in practical situations, as discussed in a later section). Using $m=4$ markers, and assuming that $t=0$ and $\mathbf{s}=\mathbf{p}$, one finds that $\sigma_{\hat{\mathrm{s}} \perp \mathrm{n}}=250 \mu \mathrm{m}, \sigma_{\hat{\imath}}=18 \mu \mathrm{m}, \sigma_{\hat{\mathrm{n}}}=2.9^{\circ}$, and $\sigma_{\hat{\theta}}$ $=0.20^{\circ}$. Thus, the direction error is about half the rotation magnitude!

\section{THE PLANAR CASE: CENTROID}

Even though biological joint movement is generally three-dimensional, the instrumental complexities of spatial measurement warrant consideration of the planar movement case. Many authors have addressed the planar centroid in the major 'plane' of movement; see Panjabi (1979), Panjabi et al. (1982a, b), and Bryant et al. (1984) for recent results and a survey of the earlier literature.

Most of this work relies on the method of Reuleaux (1963) for finite centroid reconstruction, where the point of intersection of the midperpendiculars on two distinct landmark displacement vectors is assessed (Fig. 5). Panjabi and his co-workers have generalized this method by averaging the points of intersection for all possible combinations of such midperpendiculars,

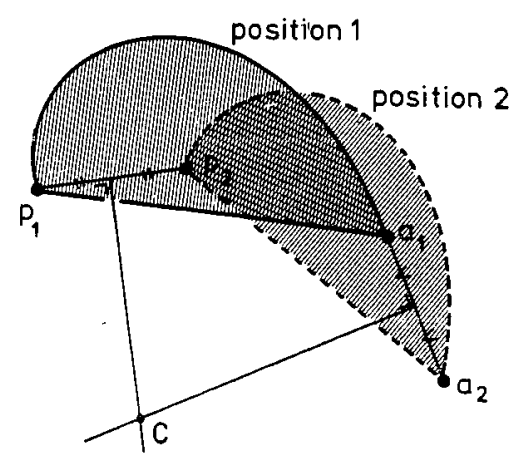

Fig. 5. Graphical centroid construction via the Reuleaux method. 
for the case of more than two landmarks. They found that the centroid's position accuracy varies inversely with the sine of half the rotation angle, and that both the rotation and centroid position errors depend on the landmark distribution.

A number of criticisms can be leveled against the Reuleaux method and its generalizations. In the first place, the Reuleaux method does not exploit the rigidbody nature of the movement: in the midperpendicular reconstruction, no use is made of the fact that the distances between the landmarks are invariant under the displacement. In the second place, the non-linear transformation from finite landmark displacements to a centroid position results in non-linearity biases which may assume significance with respect to the stochastic errors once a large number of such estimates are averaged. As apparent from Panjabi (1979) and Bryant et al. (1984), the centroid error distribution on the Reuleaux method may become quite asymmetric, and the sample mean may become a significantly biased estimate of the true centroid. Finally, in the generalization proposed by Panjabi and his coworkers, centroids from all possible combinations of two landmarks are averaged, either weighted or unweighted. This approach is statistically suboptimal since the various individual centroids are not uncorrelated, due to the repeated use of individual landmarks in the formation of all possible landmark pairs.

An approach which avoids these various drawbacks can be derived along similar lines as in the spatial case of the helical axis. Thus, the position and attitude variables of the rigid body before and after the displacement are assessed from the noisy landmark coordinates, and the rigid-body variables are subsequently used for estimating the centroid variables.

It has not been found possible to treat the planar and spatial cases in terms of a single, unified model; however, the final results exhibit considerable similarities.

\section{Position and attitude}

We shall use the same terminology and symbols as in the spatial case, but the various quantities are now planar. However, the multiplicative error attitude matrix is now replaced by an additive, scalar term $\Delta \phi_{i}$, since planar attitudes can be described by means of a single angle $\phi_{i}$.

Let $R_{i}$ be defined in terms of a single attitude angle $\phi_{i}$ between corresponding axes of $E^{\boldsymbol{x}}$ and $E^{y}$,

$$
\mathbf{y}_{i k}=\mathbf{p}_{i}+R_{i} \mathbf{x}_{k}, \quad R_{i}=\left(\begin{array}{rr}
\cos \phi_{i}, & -\sin \phi_{i} \\
\sin \phi_{i}, & \cos \phi_{i}
\end{array}\right) .
$$

Veldpaus et al. (1984) have provided an explicit leastsquares estimator for $\mathbf{p}_{i}$ and $R_{i}$. Through a linearized error propagation analysis similar to the one presented for the spatial case, and assuming zers-mean, isotropic, uncorrelated measurement errors $\Delta \mathbf{y}_{i k}$ with constant standard deviation $\sigma$ per co-ordinate as in (4), the rigidbody parameter errors are also found to be uncor- related and isotropic,

$$
E\left(\Delta \mathbf{p}_{i} \Delta \mathbf{p}_{j}^{\prime}\right)=\frac{\sigma^{2}}{m} \delta_{i j} I ; \quad E\left(\Delta \phi_{i} \Delta \phi_{j}\right)=\frac{\sigma^{2}}{m \rho^{2}} \delta_{i j}
$$

with

$$
\rho^{2} \hat{=} \frac{1}{m} \sum_{k=1}^{m} \mathbf{x}_{k}^{\prime} \mathbf{x}_{k}
$$

Here, $\rho$ is the effective (planar) landmark distribution radius. Relation (28b) differs from (1-2) in that attitude dependency via a dyadic product $\mathbf{x}_{k} \mathbf{x}_{k}^{\prime}$ does not occur, so the landmarks do not have to be isotropically distributed for attitude invariance to obtain. In the planar case, $\rho$ is the r.m.s. distance from the landmarks to the mean of the landmarks $x$, and not the r.m.s. distance to an arbitrary axis through $\mathbf{x}$ as in the spatial case.

\section{Centroid estimation}

For planar kinematics, the direction $\mathbf{n}$ and the shift $t$ of the spatial case become irrelevant, and the centroid $s$ follows from (14) as

$$
\mathbf{s}=\mathbf{p}+\left\{2 \tan \left(\frac{1}{2} \theta\right)\right\}^{-1} R\left(\frac{1}{2} \pi\right) \mathbf{d}
$$

where $\mathbf{p}$ and $\mathbf{d}$ are defined as in (13), and where $R\left(\frac{1}{2} \pi\right)$ is the rotation matrix which rotates the finite translation vector $\mathbf{d}$ through $\frac{1}{2} \pi \mathrm{rad}$ into the counterclockwise direction. Furthermore, the finite rotation angle $\theta$ now follows simply as

$$
\theta=\phi_{2}-\phi_{1} \text {. }
$$

Unlike the spatial angle of rotation, $\theta$ in (30) is a signed quantity. In the case of noisy measurements, estimates $\hat{\mathbf{s}}$ and $\hat{\theta}$ may be assessed by substituting estimates $\hat{\mathbf{p}}_{i}$ and $\hat{\phi}_{i}$ for the true but unknown quantities in (13) and (29-30). As indicated before, this approach minimizes the influence of strong non-linearities, since additive measurement errors $\Delta \mathbf{y}_{i k}$ are averaged out prior to such a transformation; this applies in particular to the centroid $\hat{\mathbf{s}}$.

\section{Centroid error propagation analysis}

The influence of the measurement errors on the centroid estimates can be analysed in the same way as done before in the spatial case. The mean position $p$ and the translation vector $d$ are modelled as additively disturbed with zero-mean errors $\Delta \mathbf{p}$ and $\Delta \mathbf{d}$ which are uncorrelated with each other and with the attitude errors $\Delta \phi_{i}$, and their covariance matrices have the same form as those in the spatial case (17). The variance of the finite rotation angle $\theta$ now follows from (28) and (30), and the covariance matrix of the centroid estimate $\hat{\mathbf{s}}$ follows by adding the contributions due to $\Delta \mathbf{p}, \Delta \mathbf{d}$, and $\Delta \theta$, by virtue of the lack of correlation between these error components. As in the spatial case, the total position variance over all directions follows from the 
trace of the centroid's covariance matrix. This yields

$$
\begin{gathered}
\sigma_{\hat{\theta}}^{2}=\frac{2 \sigma^{2}}{m \rho^{2}} \\
\sigma_{\hat{\mathrm{s}}}^{2}=\frac{\sigma^{2}}{m} \sin ^{-2}\left(\frac{1}{2} \theta\right)\left\{1+\frac{1}{2} \cos ^{-2}\left(\frac{1}{2} \theta\right)|\mathbf{s}-\mathbf{p}|^{2} / \rho^{2}\right\} .
\end{gathered}
$$

These formulae are identical to those for the spatial case $(22,25)$ if the helical shift is zero $(t=0)$. This result is quite interesting since the planar and spatial configurations are different, even in the case of planar movement in three-dimensional space.

Thus, the same conclusions can be drawn on geometrical and kinematical error sensitivities for $\hat{\theta}$ and $\hat{s}$. The error of the rotation angle is not affected by its magnitude, but it varies inversely with the landmark distribution radius. The centroid's position error varies inversely with the rotation angle magnitude, and it increases with the distance to the landmark distribution, relative to the landmark distribution radius.

The centroid is optimally determined if $\mathbf{s}=\mathbf{p}$, i.e., if it coincides with the mean position of the landmarks. The same was found by Panjabi and co-workers. Substituting $\mathbf{s}=\mathbf{p}$ in (31b), the standard deviation becomes

$$
\sigma_{\hat{\mathbf{s}}}=\frac{\sigma}{\sin \left(\frac{1}{2}|\theta|\right) \sqrt{m}} .
$$

This value is a factor $\sqrt{2}$ smaller than its counterpart at the end of the Appendix in Panjabi et al. (1982a), which must be attributed to the use of the rigid-body constraints and of statistically optimal estimation procedures. For non-optimal configurations, i.e. $\mathbf{s} \neq \mathbf{p}$, (31b) is even more favourable since determinacy obtains unless $\sin \left(\frac{1}{2} \theta\right)$ or $\rho$ vanish. According to formula (5) in Panjabi et al. (1982a), the value for $\sigma_{\hat{\mathbf{s}}}$ in the Reuleaux method $(m=2)$ becomes

$$
\sigma_{\hat{\mathbf{s}}}=\frac{\sigma}{\sin \left(\frac{1}{2}|\theta|\right) \sin \eta}
$$

where $\eta$ is the angle subtended on the centroid by the two landmarks; it is also the angle at which the two midperpendiculars intersect. This angle does not figure in the present derivations, and $\sin \eta$ may, in fact, assume arbitrary values between 0 and $2 \rho R /\left(\rho^{2}+R^{2}\right)$, where $R \hat{=}|\mathbf{s}-\mathbf{p}| /\left|\cos \left(\frac{1}{2} \theta\right)\right|$. It appears that the finite Reuleaux method yields a value for $\sigma_{\hat{\mathrm{s}}}^{2}$ which at best approaches (31b), for large values of $R / \rho$. For small $R / \rho$, the Reuleaux method always yields a much larger variance, especially for small values of $\sin \eta$.

The Reuleaux method has of ten been used for rather small values of $\sin \eta$, and this may explain to some extent the difficulties in reproducing the centroid locus within and across experiments. The present approach has certain statistical advantages: estimation biases and standard deviations for the centroid are smaller, while the results are the same for the finite rotation angle. In addition, the centroid may be reconstructed graphically for $m=2$, as is the case for the Reuleaux

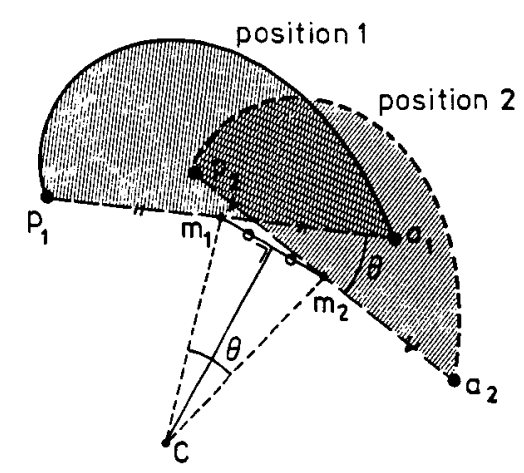

Fig. 6. Graphical centroid construction via the planar rigidbody model.

method. As shown in Fig. 6, one can draw the midperpendicular through $\mathbf{p}$ on $\mathbf{d}$, and, taking the sign of $\theta$ into account, transfer the finite rotation angle in order to delineate the centroid on this single midperpendicular. If $d$ happens to be very small, one may take the midperpendicular on the largest landmark displacement.

A word of caution: the biomechanical literature contains a large number of centroid publications in which it is merely assumed that the planar approximation based on monoscopic Röntgen photographs is viable. Such assumptions should be validated, and the present rigid-body approach allows verification of whether measured interlandmark distances are sufficiently invariant under finite displacements.

\section{IN VITRO ASSESSMENT OF HELICAL ERRORS}

In order to illustrate the theory of this paper, some of the in vitro data of De Lange et al. (in press a) were reanalyzed, by comparing the finite helical axes calculated from repeated manual digitizations of two pairs of Röntgenphotographs. The first pair of photographs was taken before, the second after a finite displacement of a wrist joint specimen.

\section{Method and results}

The radius and carpal bones of a $57 \mathrm{yr}$ old female were implanted with 3-6 tantalum pellets $(0.5-1 \mathrm{~mm}$ diameter) each. The measurement system had its $X$ axis perpendicular to the photograph cassette, parallel to the joint's antero-posterior direction, and the $Y$ - and $Z$-axes parallel to the cassette, with the $Z$-axis parallel to the longitudinal direction (see Figs 7 and 8). The radius was mounted rigidly with respect to the measurement apparatus, and the hand was moved in finite palmar-flexion rotation (i.e. about the $X$-axis) through approximately $6^{\circ}$ about the neutral attitude.

Röntgen stereopictures were taken on two pairs of photographs, one-pair before, and one pair after the displacement. The photographs were exposed to radiation from two Röntgenfoci which were $1 \mathrm{~m}$ apart and at $1.2 \mathrm{~m}$ from the photograph cassette. The carpal bones of the wrist joint were at approximately $0.1 \mathrm{~m}$ from the cassette, surrounding the midperpendicular 


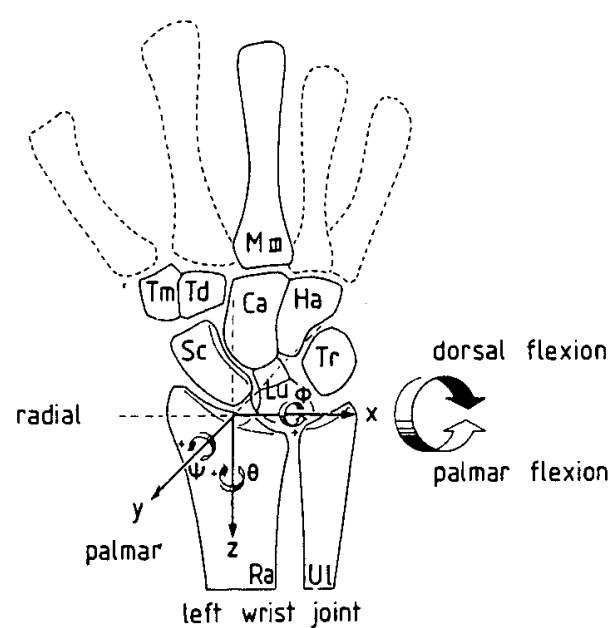

Fig. 7. Wrist joint co-ordinate systems.

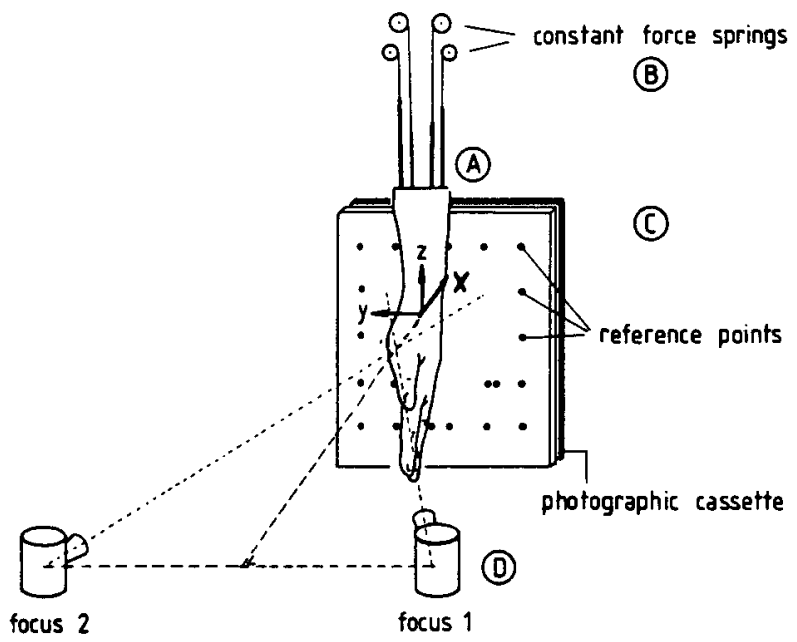

Fig. 8. Röntgenphotogrammetric measurement configuration.

from the base line between the two foci onto the cassette.

The photographs were developed and manually digitalized on a two-dimensional digitizer* with experimentally verified pellet image digitization repeatability between 10 and $15 \mu \mathrm{m}$ (standard deviation per image co-ordinate). The measurement system was calibrated, and the image co-ordinates were processed with the software package developed by Selvik (see Selvik, 1978, 1983; Van Dijk et al., 1979).

First, individual landmark co-ordinates were reconstructed via photogrammetrical triangulation for each stereophotograph individually; secondly, a rigidbody model was fitted to corresponding sets of landmarks per bone between the two photographic exposures. In both steps, redundant data were available, and optimization occurred in terms of an unweighted least-squares criterion. Finally, the helical parameters were estimated from the rigid-body data by means of an algorithm similar to the one used in the present analysis.

This sequence of numerical operations was performed for each of the two digitizing replications, and this resulted in the data shown in Table 1 . Here, $\hat{\theta}$ is the finite rotation angle, $\hat{\mathbf{n}}$ the helical axis' unit direction vector in the global co-ordinate system, and $\hat{\beta}$ the angle between the two estimated direction vectors (helical shift and position were omitted from this analysis). As apparent from Table 1, the finite rotation angle is relatively well determined, while the direction data are quite noisy.

\section{Theoretical evaluation}

The errors in Table 1 can be largely explained through the theoretical analysis of this paper. In particular, one may investigate the utility of the least-squares criterion for the various estimation phases. For the individual points, this criterion is statistically optimal insofar as the errors in the image data are predominantly caused by the digitization process, with individual errors additive, zero-mean, uncorrelated, isotropic, and having a constant standard deviation $\sigma$ per coordinate. However, it is shown below that the errors in the reconstructed landmark co-ordinates become strongly anisotropic for the given measurement configuration, and this renders the use of an unweighted

Table 1. Helical rotation and direction estimates for two digitizing replications (no. 1,2). The finite rotation angles $\hat{\theta}_{i}$, the discrepancy $\Delta \hat{\theta}=\hat{\theta}_{1}-\hat{\theta}_{2}$, and the direction error $\hat{\boldsymbol{\beta}}=\arccos \left(\hat{\mathbf{n}}_{1}^{\prime} \hat{\mathbf{n}}_{2}\right)$ are in degrees; the direction vectors $\hat{\mathbf{n}}_{i}$ are dimensionless, and of unit length

\begin{tabular}{lcccrccc}
\hline Segment & No. & $\hat{\theta}_{\boldsymbol{i}}$ & $\hat{n}_{\boldsymbol{X} \boldsymbol{i}}$ & \multicolumn{1}{c}{$\hat{n}_{\boldsymbol{Y}}$} & \multicolumn{1}{c}{$\hat{n}_{\boldsymbol{Z} \boldsymbol{i}}$} & \multicolumn{1}{c}{$\Delta \hat{\theta}$} & $\hat{\beta}$ \\
\hline Lunate & 1 & 3.48 & 0.928 & 0.060 & 0.368 & 0.43 & 15.85 \\
& 2 & 3.05 & 0.905 & -0.215 & 0.367 & & \\
Capitate & 1 & 6.73 & 0.983 & -0.068 & 0.173 & -0.29 & 3.40 \\
& 2 & 7.02 & 0.980 & 0.003 & 0.198 & & \\
Scaphoid & 1 & 6.00 & 0.961 & -0.016 & 0.277 & -0.70 & 12.92 \\
& 2 & 6.70 & 0.949 & -0.232 & 0.213 & & \\
Trapezium & 1 & 7.47 & 0.985 & -0.112 & 0.133 & 0.94 & 10.86 \\
& 2 & 6.53 & 0.989 & 0.080 & 0.127 & & \\
Trapezoid & 1 & 6.82 & 0.995 & -0.039 & 0.095 & -0.13 & 16.08 \\
& 2 & 6.95 & 0.976 & 0.219 & -0.018 & & \\
Hamate & 1 & 5.84 & 0.957 & -0.042 & 0.288 & -0.55 & 1.48 \\
& 2 & 6.39 & 0.964 & -0.067 & 0.258 & & \\
\hline
\end{tabular}

*Aristo $104-\mathrm{S}^{\circledR}$, equipped with an Aaton ${ }^{\circledR}$ video camera. 
least-squares criterion statistically suboptimal for the rigid-body estimation phase.

By means of linearized error propagation calculus, the a posteriori covariance matrix of the reconstructed landmark co-ordinates may be assessed as follows (c.f. Woltring, 1980). The collinearity between a Röntgen focus with position $\mathbf{X}_{c i}$, landmark with position $\mathbf{X}$, and image point with co-ordinates $\mathbf{x}_{i} \hat{=}\left(0, y_{i}, z_{i}\right)$, is described by the projective equation

$$
\mathbf{X}-\mathbf{X}_{c i}=\lambda_{i}\left(\mathbf{x}_{i}-\mathbf{X}_{c i}\right)
$$

where $\lambda_{i}$ is a proportionality constant. Elimination of this constant results in the collinearity equations of photogrammetry,

$\left.\begin{array}{c}X_{c i}\left(Y-Y_{c i}\right)+\left(y_{i}-Y_{c i}\right)\left(X-X_{c i}\right)=0 \\ X_{c i}\left(Z-Z_{c i}\right)+\left(z_{i}-Z_{c i}\right)\left(X-X_{c i}\right)=0\end{array}\right\} i=1,2$.

Since the calibration took place on a highly redundant set of data, calibration errors in the $\mathbf{X}_{c i}$ may be considered to be negligible. Taking the partial derivatives of (35) with respect to $\mathbf{X}$ and $\mathbf{x}_{i}$, followed by elimination of $\mathbf{x}_{i}$ results in the following error propagation relation between image errors $\Delta \mathbf{x}_{i}$ and ensuing landmark reconstruction errors $\Delta \mathbf{X}$,

$$
\frac{X_{c i}}{X_{c i}-X}\left|\begin{array}{l}
-\left(Y-Y_{c i}\right) /\left(X-X_{c i}\right), 1,0 \\
-\left(Z-Z_{c i}\right) /\left(X-X_{c i}\right), 0,1
\end{array}\right| \Delta \mathbf{X}=\left|\begin{array}{c}
\Delta Y_{i} \\
\Delta z_{i}
\end{array}\right| .
$$

For the given geometry with $\mathbf{X}_{c i}=(-1.2 \mathrm{~m}$, $\pm 0.5 \mathrm{~m}, 0 \mathrm{~m})^{\prime}$, merging of equations (36) over both foci yields, for a point at the centre of the wrist joint with true co-ordinates $X=(-0.1 \mathrm{~m}, 0 \mathrm{~m}, 0 \mathrm{~m})^{\prime}$,

$$
\begin{aligned}
B \Delta \mathbf{X} & =\Delta \mathbf{x}, \quad B \cong \frac{12}{11}\left|\begin{array}{ccc}
+1 / 2.2, & 1, & 0 \\
0, & 0,1 \\
-1 / 2.2, & 1, & 0 \\
0, & 0,1
\end{array}\right|, \\
\Delta \mathbf{x} & =\left|\begin{array}{l}
\Delta y_{1} \\
\Delta z_{1} \\
\Delta y_{2} \\
\Delta z_{2}
\end{array}\right| .
\end{aligned}
$$

Under the given error assumptions, $E(\Delta \mathbf{x})=0$, and $\operatorname{cov}(\Delta \mathbf{x})=\sigma^{2} I$. The covariance matrix of a leastsquares estimate $\mathbf{X}$ for $\mathbf{X}$ now follows as

$$
\operatorname{cov}(\Delta X)=\sigma^{2}\left(B^{\prime} B\right)^{-1}=\frac{121}{288} \sigma^{2} \operatorname{diag}\left(2.2^{2}, 1,1\right)
$$

This result shows that the errors in the reconstructed landmark co-ordinates, although uncorrelated, are certainly not isotropic. For $\sigma=10 \mu \mathrm{m}$, the errors in $Y$ and $Z$ have a standard deviation of $6.5 \mu \mathrm{m}$, and the errors in $X$ one of $14.3 \mu \mathrm{m}$. Thus, unweighted leastsquares estimation of the rigid-body parameters is statistically suboptimal, for the present measurement configuration.

Also by linearized error propagation analysis, the covariance matrix of the rigid-body parameters may be assessed, using a generalized form of the theoretical results of this paper. Since the true co-ordinates of the landmarks with respect to some body-fixed coordinate system $E^{x}$ were not known, they were approximated with the reconstructed co-ordinates of the first stereophotograph, for each bone. Thus, $\hat{\mathbf{y}}_{1 k} \hat{=} \mathbf{x}_{k}$, with $k=1, \ldots, m$, and this entails that $\hat{\mathbf{p}}_{\mathbf{1}}=\mathbf{0}$ and $\hat{R}_{1}=I$ (note that these are estimated values, since the $\left\{\hat{\mathbf{y}}_{1 k}\right\}$ are noisy). Furthermore, since the landmarks are relatively close to each other in terms of the geometry of the measurement configuration, it is reasonable to assume that all reconstructed landmark co-ordinates have the same covariance matrix (38).

Linearized covariance propagation based on relation (38) has been found to result in the approximate standard deviations for the rotation angles and direction errors listed in Table 2 , under the assumption that $\sigma=10 \mu \mathrm{m}$ per image co-ordinate. The standard deviations are different between replications since the $\left\{\hat{\mathbf{y}}_{1 k}\right\}$ are different between replications, and they are usually much smaller than the discrepancies $|\Delta \hat{\theta}|$ and $\hat{\beta}$ in Table 1. This suggests the influence of unmodelled errors since one would expect that, on the average, the discrepancies of Table 1 are merely a factor $\sqrt{2}$ larger, assuming no correlation between the errors in the two digitized replications.

One such error source is the statistical suboptimality of the unweighted least-squares criterion for rigidbody estimation, particularly if the landmarks are predominantly co-planar and parallel to the $Y Z$-plane: in this situation, the $X$-errors have the largest influence on rotational precisions. Other factors may be caused by limitations of the photogrammetrical model. For example, the Röntgen foci are not infinitesimally small, and this may entail increased blurring of pellet images with increasing distance between the pellets and the photographic plate, thus causing an effective increase in $\sigma$.

For the present rotational data, alignment errors between the two pairs of stereophotographs are not expected to be a significant error source. This alignment occures via a rigid-body fit to commonly observed fiducial markers on the photographic cassette, with an effective marker distribution radius of $100 \mathrm{~mm}$. The landmark distribution radii of the carpal bones are much smaller, of the order of $5 \mathrm{~mm}$. However, translation errors in the alignment might

Table 2. Analytical, approximate estimates for the standard deviations of the finite rotation angle $\hat{\theta}_{i}$ and of the direction error $\alpha_{i} \hat{=} \alpha_{\hat{\mathrm{n}}_{i}}$ (cf. Fig. 4), for two digitizing replications (all values are in degrees)

\begin{tabular}{lcccc}
\hline Segment & $\hat{\sigma}_{\hat{\theta} 1}$ & $\hat{\sigma}_{\hat{\alpha} 1}$ & $\hat{\sigma}_{\hat{\partial} 2}$ & $\hat{\sigma}_{\hat{\alpha} 2}$ \\
\hline Lunate & 0.075 & 1.733 & 0.065 & 2.100 \\
Capitate & 0.049 & 0.663 & 0.050 & 0.634 \\
Scaphoid & 0.081 & 1.434 & 0.107 & 1.137 \\
Trapezium & 0.433 & 4.127 & 0.340 & 5.252 \\
Trapezoid & 0.114 & 1.264 & 0.103 & 1.302 \\
Hamate & 0.171 & 2.027 & 0.172 & 1.842 \\
\hline
\end{tabular}


have assumed significance since these are merely determined by the number of fiducial markers, and not by their distribution.

\section{DISCUSSION}

In order to arrive at analytically tractable formulae, the theory of this paper was based on a number of simplifying assumptions such as isotropic conditions for the measurement errors and for the spatial landmark distribution. In practice, these conditions are rarely met, and the error sensitivities are much more complicated functions of the geometrical and kinematical situation. Nevertheless it is believed that the same general conclusions hold: the finite rotation angle and the helical shift are relatively well determined, while the direction and position of the helical axis and centroid are very sensitive to landmark measurement errors, particularly in the cases of small rotations, large distances to the mean centre of gravity of the landmarks, and small landmark distribution sizes.

If interest is directed to relative movement between adjacent rigid bodies, and neither body is fixed with respect to the measurement apparatus, the error sensitivities become even more complicated functions. However, it is believed that the theoretical conclusions can also be generalized to this case: both landmark distributions should be sufficiently large and close to the anticipated loci of the helical axis or centroid in the two co-ordinate systems, and the error sensitivity in the direction and position variables will vary inversely proportional to (small) rotations.

The assumption of accurately known local coordinates $\left\{\mathbf{x}_{k}\right\}$ can rarely be met in practice. This is not a problem when estimating the helical axis or centroid in the global co-ordinate system $E^{y}$, since the local coordinates are eliminated through the operations (12) and (30); however, knowledge of the $\left\{\mathbf{x}_{k}\right\}$ is necessary if the helical axis or centroid are also to be estimated in the local coordinate system $E^{x}$. The best approach for acquiring suitable estimates for the $\left\{\mathbf{x}_{k}\right\}$ is to conduct some form of iterative, general adjustment calculus in which the rigid-body variables $\left\{\mathbf{p}_{i}, R_{i}\right\}$ for each exposure individually and the unknown co-ordinates $\left\{\mathbf{x}_{k}\right\}$ common to all exposures are simultaneously estimated for all finite displacements $i=1, \ldots, n \geqslant 2$. For some reference exposure, $\mathbf{p}_{i}$ and $R_{i}$ must then be defined a priori. A similar approach was taken for a camera calibration procedure in Woltring (1980), where certain geometrical parameters per exposure were estimated simultaneously with camera parameters common to many exposures.

In the case of a single finite displacement $(i=1,2)$, one might also equate the $\left\{\mathbf{x}_{k}\right\}$ to the $\left\{\hat{\mathbf{y}}_{1 k}\right\}$ prior to the finite displacement, as was done in the section on experimental data.

It was shown that the finite helical axis and centroid are ill-determined from noisy landmark co-ordinates under small, finite rotations. The choice of a suitable model incorporating known rigid-body constraints may cause a substantial improvement as was shown for the planar rigid-body model relative to the finite Reuleaux method, but the results remain sensitive to measurement errors under small rotations. Thus, the finite helical axis and centroid appear to be rather problematic estimators for their instantaneous counterparts.

This situation is similar to the case of estimating velocities from finite position differences. Given position estimates $\left\{\hat{x}_{i}\right\}$ with additive, zero-mean, uncorrelated, stationary noise (standard deviation $\sigma$ ) and sampling interval $\tau$, the symmetric, finite difference velocity estimator

$$
\hat{v}_{i} \hat{=}\left(\hat{x}_{i+1}-\hat{x}_{i-1}\right) /(2 \tau)
$$

has noise standard deviation $\sigma_{\hat{v}}=\sigma /(\tau \sqrt{2})$, which varies inversely with $\tau$. Furthermore, this estimator has a strong low-pass filtering character, since its transfer function $H(j \omega)$ is equal to $j\{\sin (\omega \tau) / \tau\}$, whereas a true differentiator's transfer function is equal to $j \omega$ (NB: $j \hat{=} \sqrt{-1}, \omega \hat{=} 2 \pi f$ is the circular frequency in rad s $\mathrm{s}^{-1}$, and $f$ the frequency in $\mathrm{s}^{-1}$ ). The ratio of these transfer functions provides the effective low-pass filter characteristic

$$
H_{1 p}(j \omega)=\sin (\omega \tau) /(\omega \tau)
$$

which is equal to 1 for small frequencies $(|\omega \tau| \ll 1 \mathrm{rad})$, and significantly lower than 1 for higher frequencies. However, high frequencies are not completely attenuated. For low frequencies, (39) is an unbiased velocity estimator, but strong biases and noise transmission occur at higher frequencies. The helical axis and centroid suffer from similar problems, since they are functions of the moving body's position and velocities, and since division by a small finite rotation is required for appropriate approximation of the instantaneous case by the finite case.

A large variety of better low-pass differentiation filters have been described in the literature. Similarly, there are better methods for estimating discrete samples of the instantaneous helical axis or centroid than by the direct use of their finite counterparts on raw landmark position data. In Woltring and Huiskes (in press), a continuous movement model was fitted to noisy samples of a continuous movement. The data were low-pass filtered and differentiated by means of a modified version of the optimal regularization method of Anderssen and Bloomfield (1974a, b) described by Hatze (1981), whence samples of the instantaneous helical axis were assessed. This approach resulted in a considerable noise reduction and in very small biases in the helical position and direction estimates.

Estimating derivatives from noisy data is an example of so-called ill-posed problems (e.g. Tikhonov and Arsenin, 1977). In many applied fields including computerized tomography, electrocardiography, metereology and seismology, similar ('inverse') problems of an ill-posed nature occur. Regularization theory is an actively researched topic for solving these types of problems, with connections to classical Wiener filter- 
ing. The Wiener filter (Wiener, 1949) is the optimal, linear, and stationary filter, in the minimum variance sense, for processing signals which are additively disturbed by measurement noise, for given signal and noise power spectra: cf. Tikhonov and Arsenin (1977). Further details on optimally regularizing algorithms including cubic and quintic splines are presented in Woltring (in press). One of these algorithms, the quintic spline package of Utreras (1980) has been recently used for processing wrist joint motion data: see De Lange et al. (in press a).

The use of such smoothing techniques will result in significantly improved helical and centroid estimates. Nevertheless, they will not solve the problem of noise sensitivity completely since the helical axis and centroid are undefined under pure translations. Thus, a certain amount of rotatory movement remains necessary for the numerical use of these kinematical entities.

Acknowledgements-The authors are indebted to Dr. J. Skilling, University of Cambridge, U.K., for proofreading the final manuscript, and for his comments on a number of geometrical issues.

This work is part of a greater project which is sponsored in part through Grant number $90-90$ of the Netherlands Organization for the Advancement of Pure Research (Z.W.O.).

\section{REFERENCES}

Anderssen, R. S. and Bloomfield, P. (1974a) Numerical differentiation procedures for non-exact data. Num. Math. 22, 157-182.

Anderssen, R. S. and Bloomfield, P. (1974b) A time series approach to numerical differentiation. Technometrics 16, 69-75.

Bryant, J. T., Wevers, H. W. and Lowe, P. J. (1984) One parameter model for error in instantaneous centre of rotation measurements. J. Biomechanics 17, 317-322.

Dijk, R. van, Huiskes, R. and Selvik, G. (1979) Röntgen stereophotogrammetric methods for the evaluation of the three dimensional behaviour and cruciate length patterns of the human knee joint. J. Biomechanics 12, 727-732.

Hatze, H. (1981) The use of optimally regularized Fourier series for estimating higher-order derivatives of noisy biomechanical data. J. Biomechanics 14, 13-18.

Huiskes, R., Dijk, R. van, Lange, A. de, Woltring, H. J. and Rens, Th. J. G. van (in press) Kinematics of the human knee joint. Biomechanics of Normal and Pathological Human Articulating Joints (Edited by Berme, N. and Engin, A. E.). Sijthoff and Noordhoff, Alphen aan de Rijn.

Lange, A. de, Huiskes, R., Kauer, J. M. G. and Woltring, H. J. (in press a) On the application of a smoothing procedure in the kinematical study of the human wrist joint in vitro. Selected proceedings of the 4th Meeting of the European Society of Biomechanics, Davos/Switzerland.

Lange, A. de, Kauer, J. M. G. and Huiskes, R. (in press b) The kinematic behavior of the human wrist joint: a Röntgen stereophotogrammetric analysis. Orthop. Res.

Lesh, M. D., Mansour, J. M. and Simon, S. R. (1979) A gait analysis subsystem for smoothing and differentiation of human motion data. J. biomech. Engng 101, 205-212.

Mikhail, E. (1976) Observations and Least-Squares. IEP/Dun Donnelley, New York.

Panjabi, M. M. (1979) Centers and angles of rotation of body joints: a study of errors and optimization. J. Biomechanics 12, 911-920.

Panjabi, M. M., Goel, V. K. and Walter, S. D. (1982a) Errors in kinematic parameters of a planar joint: guidelines for optimal experimental design. J. Biomechanics 15, 537-544.

Panjabi, M. M., Goel, V. K., Walter, S. D. and Schick, S. (1982b) Errors in the center and angle of rotation of a joint: an experimental study. J. biomech. Engng 104, 232-237.

Pezzack, J. C., Norman, R. W. and Winter, D. A. (1977) An assessment of derivative determining techniques used for motion analysis. J. Biomechanics 10, 377-382.

Reuleaux, F. (1963) The Kinematics of Machinery: Outline of a Theory of Machines. Dover, New York (Translated from the original German edition of 1875).

Selvik, G. (1978) Röntgen stereophotogrammetry in Lund, Sweden. Applications of Human Biostereometrics (Edited by Coblenz, A. M. and Herron, R. E.), Proc. SPIE 166, pp. 184-189.

Selvik, G. (1983) Röntgen stereophotogrammetry in orthopaedics. Biostereometrics '82 (Edited by Herron, R. E.), Proc. SPIE 361, pp. 178-185.

Soudan, K., Audekercke, R. van and Martens, M. (1979) Methods, difficulties, and inaccuracies in the study of human joint kinematics and pathokinematics by the instant axis concept. Example: the knee joint. J. Biomechanics $12,27-33$.

Spoor, C. W. (1984) Explanation, verification and application of helical-axis error propagation formulas. Hum. Mvmt. Sci. 3, 95-117.

Spoor, C. W. and Veldpaus, F. E. (1980) Rigid body motion calculated from spatial co-ordinates of markers. J. Biomechanics 13, 391-393.

Tikhonov, A. N. and Arsenin, V. Y. (1977) Solutions of Illposed Problems. John Wiley, New York.

Utreras, F. (1980) Un paquete de programas para ajustar curvas mediante funciones spline. Informe Tecnico MA-80B-209, Departamento de Matematicas, Faculdad de Ciencias Fisicas y Matematicas, Universidad de Chile, Santiago/Chile.

Veldpaus, F. E., Woltring, H. J. and Dortmans, L. J. G. M. (in press) A least-squares algorithm for the equiform transformation from spatial marker co-ordinates.

Wiener, N. (1949) The Extrapolation, Interpolation and Smoothing of Stationary Time Series. John Wiley, New York.

Woltring, H. J. (1980) Planar control in multi-camera calibration for three-dimensional gait studies. J. Biomechanics 13, 39-48.

Woltring, H. J. (1982) Estimation and precision of threedimensional kinematics by analytical photogrammetry. (Edited by Paul, J. P., Jordan, M. M., Ferguson-Pell, M. W. and Andrews, B. J.) Computing in Medicine. The MacMillan Press, London/Basingstoke.

Woltring, H. J. (in press) On optimal smoothing and derivative estimation from noisy displacement data in Biomechanics. Hum. Mumt. Sci.

Woltring, H. J. and Huiskes, R. (in press) A statistically motivated approach to instantaneous helical axis estimation from noisy, sampled landmark co-ordinates. Biomechanics IX (Edited by Winter, D. A. et al.). Human Kinetics Publishers, Champaign, IL. 